\title{
Using Spectrograms from Underwater Total Pressure Sensors to Detect Passing Vessels in a Coastal Environment
}

\author{
MARgus RÄtseP, KeVIn E. PARnell, AND TARMo SOOMERE \\ Department of Cybernetics, School of Science, Tallinn University of Technology, Tallinn, Estonia \\ MaArja KruusmaA, Asko Ristolainen, and JefFrey A. Tuhtan \\ Centre for Biorobotics, Department of Computer Systems, School of Information Technologies, \\ Tallinn University of Technology, Tallinn, Estonia
}

(Manuscript received 25 November 2019, in final form 11 May 2020)

\begin{abstract}
Monitoring vessel traffic in coastal regions is a key element of maritime security. For this reason, additional ways of detecting moving vessels are explored by using the unique structure of their wake waves based on pressure measurements at the seabed. The experiments are performed at a distance of about $2 \mathrm{~km}$ from the sailing line using novel multisensor devices called "hydromasts" that track both pressure and near-bed water flow current velocities. The main tool for the analysis is a windowed Fourier transform that produces a spectrogram of the wake structure. It is shown that time series from the pressure sensors, measured at a frequency of $100 \mathrm{~Hz}, 0.2 \mathrm{~m}$ above the seabed are a valid source of input data for the spectrogram technique. This technique portrays the properties of both divergent and transverse waves with an accuracy and resolution that is sufficient for the evaluation of the speed and distance of the detected vessels from the measurement device. All the detected passings are matched with vessels using automatic identification system (AIS) data. The use of several time series from synchronized multisensor systems substantially suppresses noise and improves the quality of the outcome compared to one-point measurements. Additional information about variations in the water flow in wakes provides a simple and reasonably accurate tool for rapid detection of ship passages.
\end{abstract}

\section{Introduction}

The ability to detect and identify passing vessels, their properties, and sailing parameters has a number of important applications. They include maintaining control in territorial waters (Till 2013), recognizing illegal fishing (Kurekin et al. 2019), avoiding navigational accidents (Chen et al. 2018), ensuring the security of various facilities (Dugad et al. 2016; Anupriya and Sasilatha 2018), and allowing a better understanding of the impact of high speed or strongly powered ships on the coastal environment (Soomere et al. 2011).

Several techniques can be used to detect the presence of vessels and distinguish and monitor vessel movements. The most common are radar and radio surveillance (Siegert et al. 2019), satellite sensing including synthetic aperture (SAR) technology (Zilman et al. 2004; Gierull 2019; Renga et al. 2019), both airborne and local

\footnotetext{
Corresponding author: Margus Rätsep, margus.ratsep@ttu.ee
}

(visual and hyperspectral) optical techniques (Park et al. 2018), various acoustic (sonar) technologies (Huang et al. 2017; Zhu et al. 2018), and classic visual observations from the coast or patrolling vessels.

Some of these techniques (e.g., several acoustic recognition systems) are only able to detect the presence of a ship in a certain region. More advanced technologies provide, similarly to the automatic identification system (AIS; self-reporting system on board of ships for vessel tracking), the location (or a sequence of locations) of vessels. However, the detection of the sailing parameters (speed and the course of the vessel) from such data sources is not always possible (Fujino et al. 2018). Also, none of these methods guarantees the reliability of detection of vessels present in a given region under every possible scenario. To address the gaps in both reliability and accuracy, additional means for the surveillance of sea areas should be studied.

A feasible way forward is to improve the accuracy of detection of disturbances that a ship creates in the 
surrounding environment (Panico et al. 2017). A moving ship always generates a system of surface waves called a wake. From about three ship lengths behind every displacement vessel, a characteristic wake emerges (Newman 1977). The properties of its main components have been known for decades (Sorensen 1973; Wehausen 1973). It usually consists of the turbulent wake (seen mostly as foam behind the ship) (Zilman et al. 2004; Fang et al. 2011) and the Kelvin wave wake (Fig. 1) (Sorensen 1973; Wehausen 1973).

The Kelvin wake consists of two wave systems. The crests of longer transverse waves form a large angle with respect to the ship's track and almost follow the ship's motion. The crests of shorter divergent waves are roughly parallel to the ship's path. These waves propagate mostly out of the wave generation area (Sorensen 1973; Wehausen 1973). This characteristic triangular spatial pattern of ship wakes (Fig. 1) is implemented, e.g., in the analysis of the data from satellite photography and synthetic aperture radar, for vessel detection (Zilman et al. 2004).

Vessel wakes have also unique structure in the spectral representation of the wave elevation data (Wyatt and Hall 1988; Sheremet et al. 2013; Torsvik et al. 2015; Pethiyagoda et al. 2017, 2018). Moreover, it is possible to extract different wake components from this representation to characterize the movement of the vessel (Torsvik et al. 2015; Pethiyagoda et al. 2017).

Field experiments have used two sources of time series to characterize the ship wake. High-frequency tracking of water surface undulations created by wakes using downwardlooking devices were used by Didenkulova et al. (2013) and Torsvik et al. (2015), among others. Pressure time series in the water column or on the seabed were utilized, e.g., in Sheremet et al. (2013) and Benassai et al. (2015). Both methods produced well-defined spectrograms that allowed for detailed analysis of vessel wakes (Sheremet et al. 2013; Torsvik et al. 2015). However, the spectrograms obtained from pressure recordings (Sheremet et al. 2013; Benassai et al. 2015) highlighted only the energy (or height) of ship wakes and the distribution of energy between wake components of different length, but not the properties of the transverse and divergent waves. More generally it was not possible to properly describe the wake components in the time-frequency plane in the manner that can be used for estimating vessel speed and wake traveling distance (Torsvik et al. 2015).

Both approaches have some shortcomings. The intense wind-wave background can shadow shorter components of ship wakes in the spectrogram based on water surface undulations and limit the extraction and analysis of the associated properties of ships (Torsvik et al. 2015). Also, the pressure signal of shorter waves rapidly attenuates in the water column. Therefore, the pressure readings have

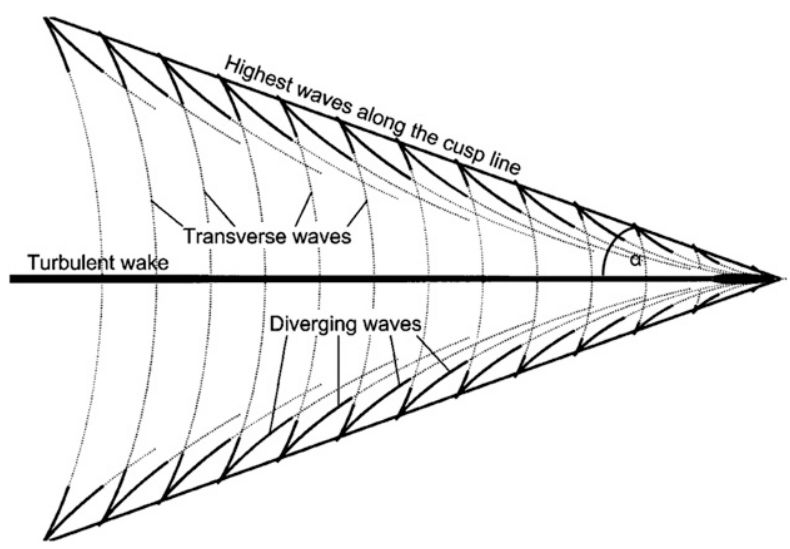

FIG. 1. The standard scheme of the classic Kelvin ship wakes; the ship is moving to the right. The half angle of the wedge is $\alpha \approx 19.47^{\circ}$.

to be corrected to compensate for the attenuation of the higher-frequency part of the wave data. As a result, some of the information about the wake may be lost (Cavaleri 1980; Kuo and Chiu 1994; Karimpour and Chen 2017).

This paper aims to extend the spectrogram-based analysis of the properties of transverse and divergent waves of vessel wakes from surface elevation recordings (Torsvik et al. 2015) to datasets recorded by pressure sensors incorporated into novel multisensor measuring devices. We start from a short discussion of the core properties of wave wakes of vessels and their reproduction in spectrograms. This is followed by a demonstration that the data collected from pressure sensors can be used to analyze the ship's movement at the same level of detail as it is done based on other types of recordings of surface elevations.

To raise the signal-to-noise ratio in wake spectrograms and improve the quality and contrast of the signatures of vessels in spectrograms, we use recordings from a gridded array of five identical pressure sensors. Finally, we discuss the potential applications and possible extensions of the presented technology for estimates of ship location, speed, and course. As ship motion always produces a wave wake, this approach could be (a part of) an automatic vessel detection system. The main message is that the pressure recordings from the seabed, even if associated with a certain loss of information, may provide a convenient prefiltered time series in which the wind-wave signal is attenuated and ship wakes are highlighted.

\section{Methodology and experimental setup}

\section{a. Kelvin wake}

The two components of the Kelvin wake, the divergent and transverse waves, are basically linear waves. 


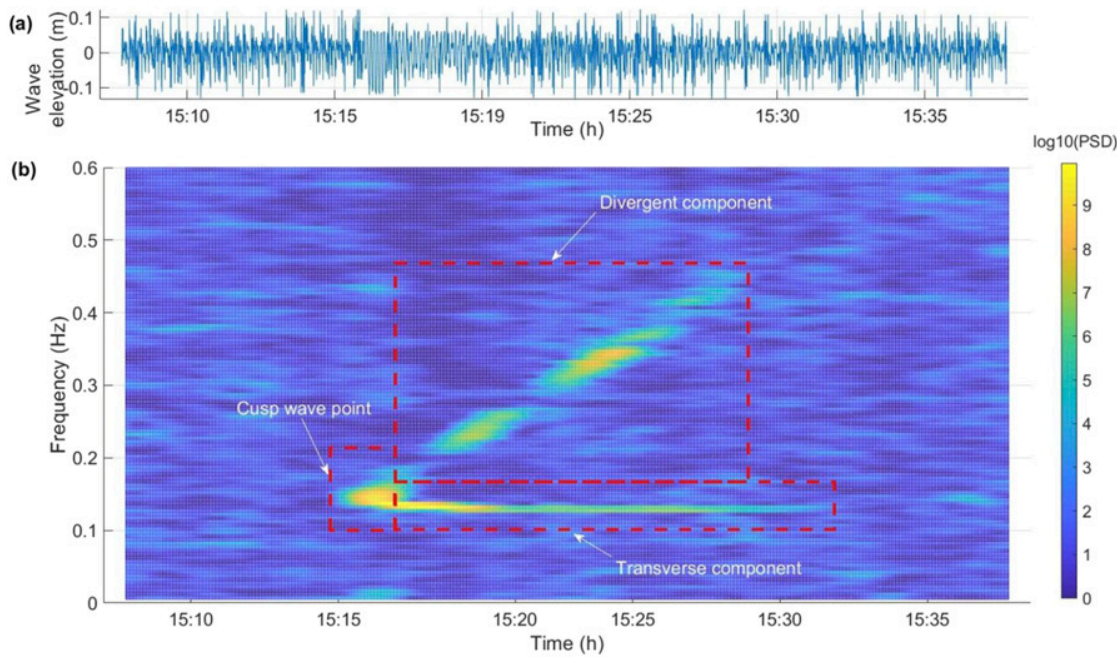

FIG. 2. (a) An example of the pressure time series of the vessel wake that is converted to water elevation data. (b) Short-time Fourier transform of the corresponding series. Wake elements are marked with red dashed rectangles.

Even though ship wakes may contain other (nonlinear) components (Soomere 2007), or have other specific features for high sailing speeds (such as features characteristic to the Mach cone; Rabaud and Moisy 2013), the divergent and transverse waves, are always present if the ship is sailing at speeds below a certain threshold called critical speed. This speed is the maximum propagation speed $\sqrt{g h}$ of long waves for the given water depth $h$ and the acceleration due to gravity $g$ (Sorensen 1973). The waves fill a triangular area called the Kelvin wedge (Fig. 1). The largest wave amplitudes (cusp waves) occur along so-called cusp lines at the border of the Kelvin wedge owing to the interaction of transverse and divergent waves (Kuznetsov et al. 2002).

In deep water, the half angle of the Kelvin wedge is $\arcsin (1 / 3) \approx 19.47^{\circ}$ (Fig. 1$)$, the shape of this wedge does not depend on the sailing speed, and the diverging waves are short crested. The signature of such wakes in the spectrogram representation has a characteristic $L$-like shape (Fig. 2b). The upper, inclined part of this signature corresponds to shorter divergent waves. It has a frequency increasing over time (also known as a chirp signal), which is commonly observed at a fixed location at the seashore after the passage of a steadily sailing ship (Sheremet et al. 2013). The lower, mostly horizontal part of this signature represents longer transverse waves that have a constant frequency for a steadily sailing ship and is also constant for a fixed observer. The cusp waves are represented by the common point of these two parts of the signature.

If the ship sails in waters where the length of excited waves is 2 times or more the water depth, the Kelvin wedge becomes wider, a large portion of the energy of generated waves is concentrated in a few divergent waves and the transverse waves become weaker (Sorensen 1973; Soomere 2007). The wave system becomes highly nonlinear and contains several other components at socalled near-critical speeds (Soomere 2007) when the sailing speed is $\pm 15 \%$ of the critical speed. At even higher speeds most of the wave energy is concentrated in a few longcrested divergent waves. This kind of wake may contain specific types of solitons (Soomere 2007) or resemble a Mach-type wave system (Rabaud and Moisy 2013). The described transformation makes it possible to identify some properties of sailing ships from the records of their wakes (Wyatt and Hall 1988) and to create characteristic "portraits" of the wake systems for different ships and for specific speeds (Torsvik et al. 2015; Pethiyagoda et al. 2018). In this paper, we focus on the extraction of the classic $L$-like signatures from the viewpoint of efficient (all passages detected) and reliable (no false signals) detection of ship passages.

\section{b. Measurement devices}

For measuring the wakes we used a device called a "hydromast" (Ristolainen et al. 2016), which is designed to sense near-bed drivers of hydrodynamic processes. Its name is inspired by the neuromast of the lateral line of the fish, which is used for sensing the flow (Bleckmann and Zelick 2009). In addition to pressure data, it incorporates the possibility of obtaining a proxy of flow speed and direction using inertial measurements of the water flow (Ristolainen et al. 2019). In this paper, we only discuss part of the functional capacity of hydromasts, namely, their pressure sensors, the option of having a proxy of flow velocities, and the ability to provide 

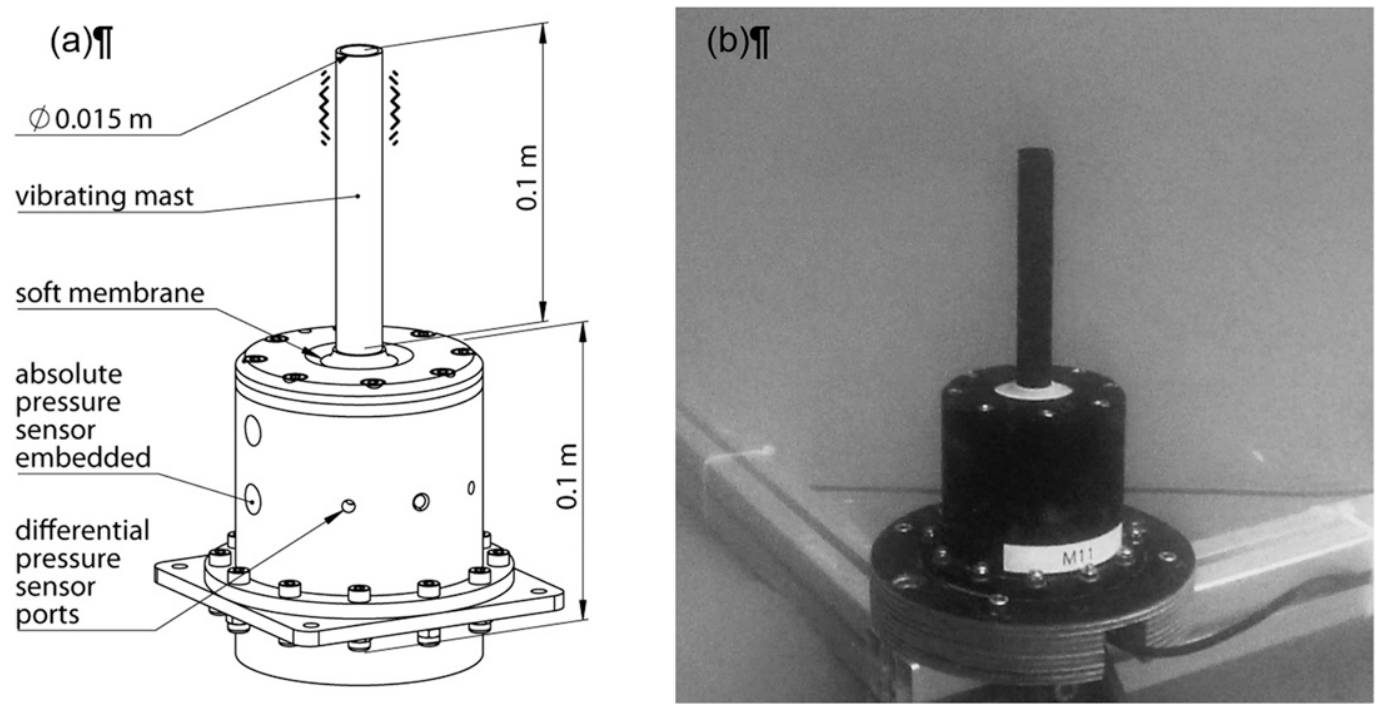

FIG. 3. (a) The components of the hydromast. (b) The working device.

synchronized pressure recordings from an array of devices.

The hydromasts used in this study (Fig. 3) encompass a vibrating stem that is fixed to a pressure-sensitive body (Ristolainen et al. 2019). Vortex induced vibrations are caused by vortex shedding from the stem. The vibrations are measured in the directions of three perpendicular axes by a micromechanical inertial measuring unit (IMU) fixed to the lower end of the stem (Fig. 3a). The device incorporates another identical, stationary, IMU, for reference and for reducing measurement noise generated by the hydromast body self-motion. The stem is made from $100 \mathrm{~mm}$ long, $15 \mathrm{~mm}$ diameter rigid hollow polyoxymethylene (POM) plastic. The aim was to have the density as close to the surrounding water as possible to reduce the restoring force caused by buoyancy. Pressure is measured using an absolute pressure sensor (MPX5100GP, NXP) for recording the water depth and two differential pressure sensors (MPXV7002, NXP) for measuring dynamic pressure relative to the stagnation point (Ristolainen et al. 2019). The output stream of the device includes inter alia a time stamp in milliseconds, the absolute and two differential pressure sensor outputs in millivolts, temperature, and calibration status.

\section{c. Field experiment}

The results presented below are based on a dataset recorded in the nearshore of Tallinn Bay. It is a semiclosed area at the southern coast of the Gulf of Finland in the northeastern Baltic Sea, with dimensions of approximately $10 \mathrm{~km} \times 20 \mathrm{~km}$. This area is known for extensive traffic of strongly powered ships that sail at or close to near-critical speeds (Soomere 2005). Most of the vessel traffic in the bay follows the traffic separation scheme for entering/departing the Port of Tallinn (Fig. 4). The typical shortest distance from the ships to the device location is about $1.5-2.5 \mathrm{~km}$. Measurements were conducted from 10 to 21 July 2017 near Pikakari Beach (Fig. 4), a small accumulation feature with a sandy and gently sloping nearshore on the western shore of Tallinn Bay. No recordings exist for 15 July due to the maintenance of the devices.

Nine hydromasts were assembled into a regularly spaced rectangular array using a $5 \mathrm{~m} \times 5 \mathrm{~m}$ aluminum frame. The time-averaged water depth at the measurement site was $3 \mathrm{~m}$. The frame was anchored to the bottom using $8 \mathrm{~mm}$ metal bars with additional weights at the corners of the frame. The stems were at the height of $0.3 \mathrm{~m}$ above the seabed. The absolute and differential pressure was read at a height of $0.2 \mathrm{~m}$ from the bed with a frequency of $100 \mathrm{~Hz}$. The data were saved as text files in 5 min blocks.

\section{d. Analysis of pressure data and velocity proxy}

The data streams from the sensors were merged into continuous $24 \mathrm{~h}$ blocks starting at midnight. The outliers (values that differed more than three standard deviations from the mean value of the pressure series) were removed. The absolute pressure data were used to identify and analyze the properties of the ship wakes. The recorded data $p^{*}$, originally given in millivolts, were converted into pressure $p(\mathrm{~Pa})$ using the calibration relation obtained from a water tank test:

$$
p=27.1208 p^{*}+4475.72 \text {. }
$$

The correlation coefficient between the raw data and the linear fit to the raw data is $R^{2}>0.9$. As Tallinn Bay has virtually no tides (Leppäranta and Myrberg 2009) and 


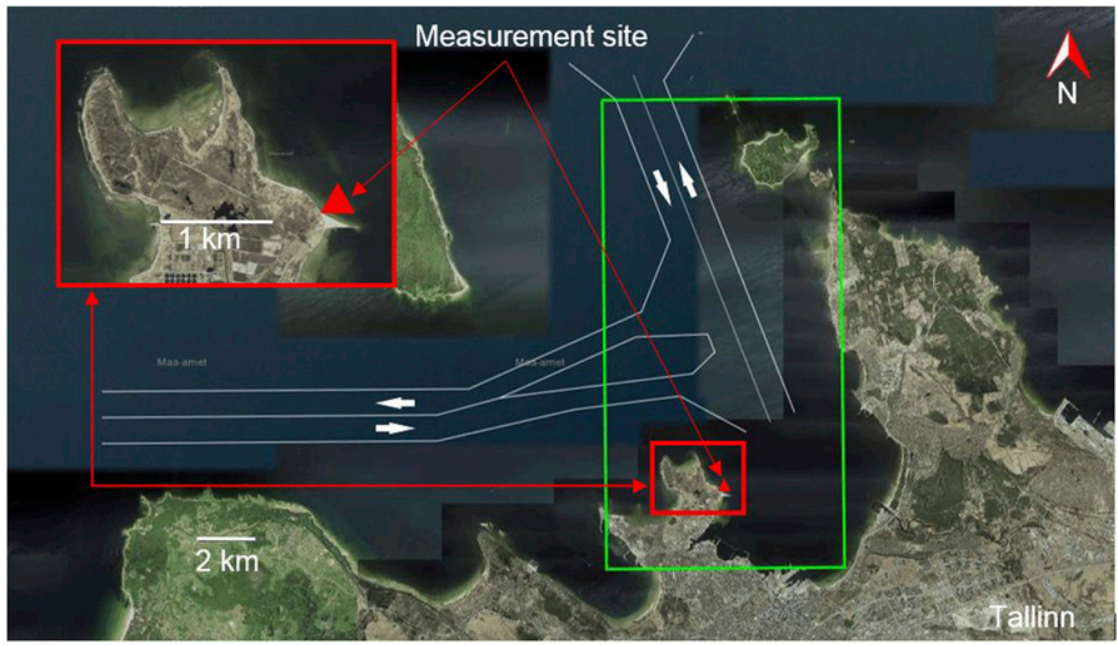

FIG. 4. The location of Tallinn Bay, traffic separation scheme (white lines), and sailing directions sailing (white arrows) along its main fairways. The measurement site is at Pikakari Beach (red triangle). The area where AIS data were retrieved is marked with a green rectangle (Estonian Maritime Administration 2019).

water level variations are just a few centimeters on calm summer days (due primarily to wind and air pressure effects), it is reasonable to assume that the water depth remained nearly constant during the fieldwork. The height of the water column above the device is the difference between the average depth $h=3 \mathrm{~m}$ at the measurement site and the height of the sensor $d_{s}=0.2 \mathrm{~m}$ above the seabed. The attenuation of the pressure signal in the water column compared to the amplitude of water surface fluctuations was corrected using the classic relationship for linear waves (Karimpour and Chen 2017):

$$
P_{0}\left(z=-h_{s}\right)=P+q=\rho g h_{s}+\rho g \eta K_{p},
$$

where $P_{0}$ is the total pressure, $P$ is static pressure (equal to the mean water pressure), $q$ is the dynamic pressure that represents the water fluctuations, $h_{s}=h-d_{s}$ is the depth of the sensor, $g$ is gravity acceleration, $\rho$ is the water density, $z$ is the depth of the sensor with positive values above the water level, $\eta$ is the water surface elevation, and $K_{p}$ is the dynamic pressure to the surface elevation conversion factor (Karimpour and Chen 2017):

$$
K_{p}=\frac{\cosh k(h+z)}{\cosh (k h)}=\frac{\cosh \left(k d_{s}\right)}{\cosh (k h)} .
$$

Here, the wavenumber $k$ is a function of the wave angular frequency $\omega=2 \pi / T$ and local water depth $h$, defined by the dispersion relation of surface gravity waves:

$$
\omega^{2}=g k \tanh (k h) .
$$

The values of the wavenumber $k$ were evaluated using the approach proposed in (Goda 2010).

The time series of fluctuations of the water surface were sampled at a frequency of $5 \mathrm{~Hz}$, which is sufficient to allow well-defined spectrograms (Torsvik et al. 2015). The short-time Fourier transform was applied to this time series with the frequency resolution of $0.005-0.6 \mathrm{~Hz}$ and a Hamming window with a length of 1024 data points. We employed an overlap of time windows of $178 \mathrm{~s}$ to obtain a good resolution with a $0.5 \mathrm{~s}$ step and to simultaneously reduce the likelihood of extracting false multiple signatures in close proximity of each other instead of one real signal. The resulting spectrograms were normalized to make it easier and more consistent to compare the results from different sensors.

Additionally, vibration level of a stem, measured by IMU could be used for automatic vessel wake detection as this information can be considered as a proxy of water velocity around the instrument. In our case, IMUs positioned in the hydromasts produced two datasets instead of one combined acceleration measurement: the linear acceleration information and gravity vector data. To detect the extreme positions of the vibrating stem, we used both of them for vessel wake detection.

For each of the hydromasts, the data from the casing IMU were subtracted from the stem IMU (Ristolainen et al. 2019). As we were only interested in very strong readings (in terms of the ratio between high and low values in the relevant time series), there was no need to convert the sensor output into dimensional units. Similar to the pressure signal, the outliers were removed. Also, both datasets were normalized and the root-mean-square 
average of the acceleration was found from the three perpendicular axes used for measurements. Highfrequency fluctuations (that cannot be part of ship wake signal) were filtered out using the ninth-order low-pass Butterworth filter with a cutoff frequency of $5 \mathrm{~Hz}$. A threshold depending on the resulting average value was applied to limit the minimum time interval between ship wakes to one event within every $10 \mathrm{~min}$. The resulting sets of possible ship wake events were compared with visual findings from the spectrograms derived from the absolute pressure data.

\section{e. The AIS data}

The signatures of wakes obtained using the pressure sensors were compared with data from the vessel AIS sourced from BigOceanData (BigOceanData 2019). The AIS data were retrieved for the entire period of fieldwork for all ships that sailed in the area marked in Fig. 4 encompassed by $59.45^{\circ}-59.60^{\circ} \mathrm{N}, 24.65^{\circ}-24.80^{\circ} \mathrm{E}$. The AIS dataset does not necessarily contain information about all ships. For example, there were no ship AIS records in the retrieved record for 19-20 July, presumably due to technical issues.

The AIS data are recorded once in every $5 \mathrm{~min}$ for all vessels. The information provided is the geographical location (longitude, latitude), the time stamp, name of the vessel, International Maritime Organization (IMO) identifier, Maritime Mobile Service Identity (MMSI) number, call sign, course, speed, and status of a vessel (BigOceanData 2019). The status can be one of the following: "undefined," "underway using engine," "underway sailing," "moored," "anchor," or "restricted maneuverability." In total 206 unique vessels were identified using a total of 82800 entries in the AIS dataset.

The data were grouped by vessels and separated into passings that may be detected at the measurement site. Passing is defined here as a closest AIS position to the measurement site from the series of consecutive AIS contacts or positions of a vessel. We only considered vessels that actually sailed in Tallinn Bay, that is, the vessels that reported their speed and course outside the harbors and anchoring areas. Most of these vessels were moving in the area of interest (Fig. 4). A single vessel may have several passings per day. The passings could represent either passage through the area of interest or relocating within the area. For example, the passenger ferry Megastar (owned and operated by Tallink) had six passings on 11 July. Megastar had three return journeys between Tallinn and Helsinki (about $60 \mathrm{~km}$ to the north of Tallinn Bay) and thus had three entries to and three departures from the Port of Tallinn. For each passing, we evaluated the closest AIS contact based on the coordinates and the closest approximate position to the location of devices based on the trajectory of the vessel.

\section{Results}

\section{a. Multiple sensors}

Experience with spectrogram analysis of surface elevation data suggests that on many occasions some components of ship wakes may be severely masked by wind waves (Torsvik et al. 2015). This is a frequent problem in semienclosed sea areas such as Tallinn Bay where even fairly moderate winds may generate waves with periods of $2-3 \mathrm{~s}$ (frequencies $0.3-0.5 \mathrm{~Hz}$ ) with a wide spectrum that overlaps with vessel wakes in frequency space. While the elimination of some features such as narrowbanded swells from the recorded signal may be possible, it is usually not feasible to remove the signal of wave fields with a wide spectrum from the spectrogram. The presence of relatively strong levels of wind-wave noise does not necessarily destroy the entire approach but may modify the results significantly and may render part of the chirp-like signal of divergent waves imperceptible in spectrograms (Torsvik et al. 2015). A typical case with such a problem is illustrated in Fig. 5a.

The use of time series pressure sensors has another shortcoming. While the high-frequency part of the surface wave field is often attenuated, the near-bed pressure signal frequently contains a substantial level of low-frequency (periods $>10 \mathrm{~s}$ ) pressure oscillations that may mask the longer components of the wake such as transverse waves or precursor solitons (e.g., near-horizontal higher energy lines up to $0.2 \mathrm{~Hz}$ in Fig. $5 \mathrm{~b}$ compared to Fig. 5a). A part of this noise has typical periods $>15 \mathrm{~s}$ and thus is much longer than ship wake components.

This shortcoming may be mitigated using several synchronized pressure sensors located at a certain distance from each other. Such a configuration is complicated and expensive to build from downward-looking devices but easily manageable using an array of hydromasts. We used for this purpose data from the five (devices on the corners and at the center of the grid) out of nine hydromasts of the frame described above. Adding data from the additional four sensors did not increase the quality of the spectrogram in terms of better signal to noise ratio. The long wave speed at the measurement location was $5.4 \mathrm{~m} \mathrm{~s}^{-1}$. The phase speed of longer vessel wake components is thus on the order of $3-4 \mathrm{~m} \mathrm{~s}^{-1}$. The phase shift of single wave crests at different sensor locations is therefore much smaller than the wave period (usually $>3 \mathrm{~s}$ ).

If the low-frequency background noise is not coherent over the distance between the sensors, it can be suppressed 

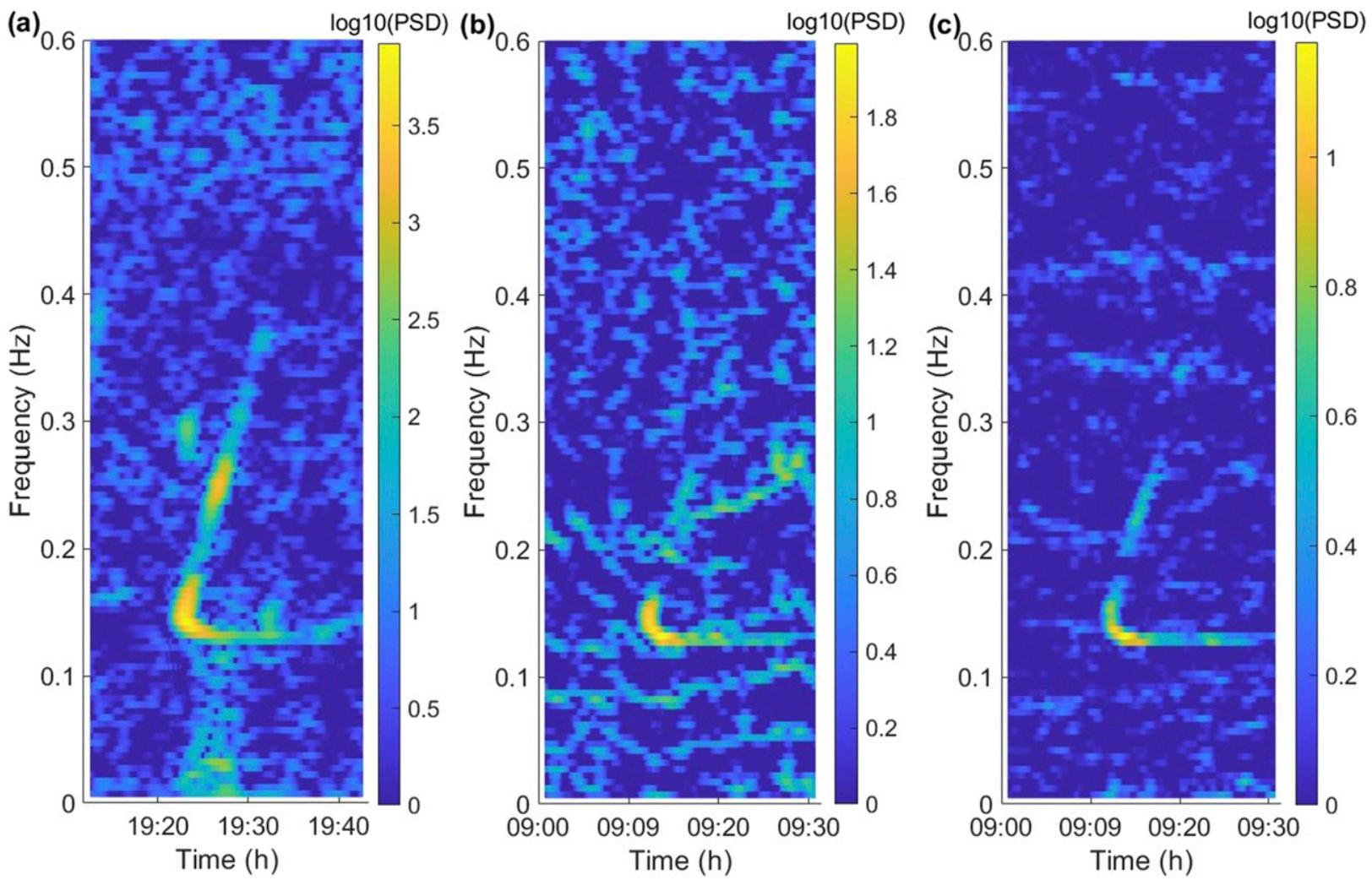

FIG. 5. (a) The signature of passenger ferry Tallink Star approaching the Port of Tallinn on 25 Jun 2009 with a wind-generated sea with typical periods of $2-2.5 \mathrm{~s}$ (Torsvik et al. 2015). The wake is measured using a one-point measurement device (a down-looking echosounder mounted on a tripod) from above the water level. (b) The signature of the same vessel approaching Port of Tallinn on $17 \mathrm{Jul} 2017 \mathrm{under}$ similar weather conditions. The wake is detected using a single pressure sensor mounted at the seabed at a depth of $3 \mathrm{~m}$. The pressure sensor is at a height of $0.2 \mathrm{~m}$ from the seabed. (c) As in (b), but visualized using five sensors located in the center and the corners of a frame of size $5 \mathrm{~m} \times 5 \mathrm{~m}$. The spectrograms are normalized by frequency spectrum and overlapped.

by merging several properly synchronized spectrogram snapshots of coherent waves into one picture. This approach was realized by using ensemble averaging, which was done by averaging the normalized spectrograms from a cluster of five sensors into a single diagram. This procedure substantially improved the wake-to-background spectrum noise ratio (Fig. 5c). It greatly simplified the wake detection process, eliminated false decisions, and made it possible to evaluate some properties of ship motion.

\section{b. Detection of a sequence of ship wakes}

The applied three-step process of detection of single wake by (i) evaluating very large values of the velocity proxy as the potential time instance of the arrival of ship wakes, (ii) subsequent spectrogram analysis of pressure fluctuations at the locations of single sensors, and (iii) refinement of the signatures of single ships by means of merging and averaging normalized spectrograms from a cluster of sensors, leads to an overall relatively efficient and reliable recognition of ship passages (Fig. 6).
An example is given in Fig. 6. During the represented time interval, 12 ship wakes can be visually identified in the spectrogram calculated from the absolute pressure data as described above (Fig. 6c). All 12 wake signatures were matched with the corresponding passings retrieved from the AIS data. Some passings have only the signature of diverging waves, which makes automatic distinguishing complicated. However, most of the detected signatures contain the visually distinguishable divergent and transverse wave components that have been used in similar studies that use wave elevation data for estimating energy, duration and spectral composition of the wake and its components (Torsvik et al. 2015). The number of ship passages found from the overthreshold values of the gravity vector data was 12 (Fig. 6b). From these events, 10 cases matched the relevant visually identified images of ship wakes in the spectrogram. Two were false-positive signals and two ship passages (at 2240 and 2350 LT; LT = UTC $+3 \mathrm{~h}$ ) were missed. The total number of ship passages identified in a similar manner from the linear acceleration dataset was 14 (Fig. 6a). From these events, 

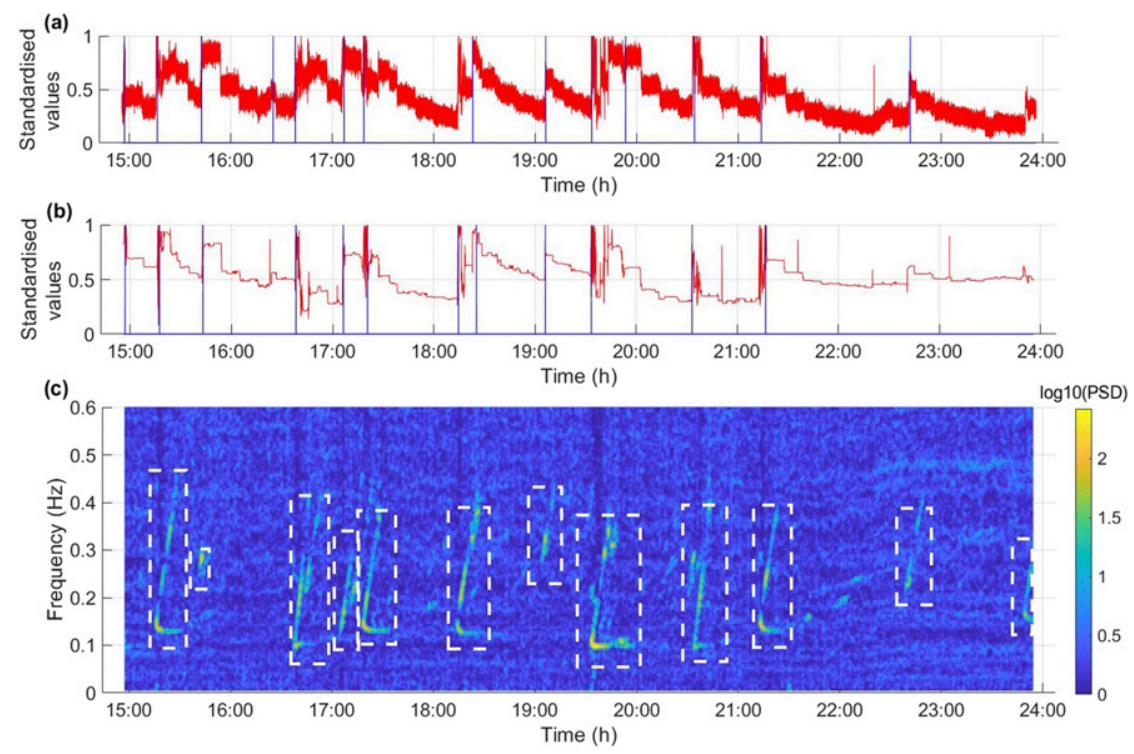

FIG. 6. The comparison of the detection results on $10 \mathrm{Jul}$ 2017. (a) Time series of the linear acceleration (red) with detected events (blue vertical lines) (the number of detected events was 14), (b) time series of the gravity vector (red) with detected events (blue) (12 wakes captured), and (c) the spectrogram based on the pressure data where ship wakes are portrayed as yellow structures (outlined with white dashed rectangles; in total 12 wakes can be seen).

11 matched the ship wake images visually identified from the spectrogram. Therefore, four contacts were false-positive and one contact was missed.

Throughout the period when both the measurement data and AIS information were present, 852 events of vessels passing by the measurement site were filtered out from the AIS data and 160 wake structures were visible in the spectrograms (as in Fig. 6c). The number of wake events in the spectrograms was limited due to the speed of the ships as wakes from the vessels sailing below $15 \mathrm{kt}$ (approximately $28 \mathrm{~km} \mathrm{~h}^{-1}$ ) were seldom visible in the spectrograms. The second factor was the location of the measurement devices, as Katariina jetty (southeast from the measurement site in Fig. 4) sheltered the measurement site from the wakes of majority of the departing vessels and the tip of Pikakari peninsula stopped wakes from the greater number of the ships operating east-west directions north from reaching the measurement site (Fig. 4). Also, on some occasions, wakes from several vessels arrived at the sensors less than $10 \mathrm{~min}$ apart, which resulted in reading them as one structure.

Due to these circumstances, we focused mainly on the vessels approaching the port of Tallinn at a speed of $15 \mathrm{kt}$ or more. There were 144 passings (out of 852) that met these criteria. Majority of them (135) were roll-onroll-off type ferries or passenger ships. The rest (9 vessels) were small craft (with a length less than $24 \mathrm{~m}$ ). From those
144 events, 116 cases were matched with corresponding wakes structures in the spectrograms, 13 misses were due to small time interval (less than $10 \mathrm{~min}$ ) between the wakes, 9 cases were small craft, and in 6 cases ferries were sailing near $15 \mathrm{kt}$.

From the 160 cases when a wake was visible in the spectrogram, 152 wakes were also detected by using the linear acceleration data and 134 by utilizing gravity vector information from the sensors. Despite the slightly better detection rate, linear acceleration information provided a higher total number of extreme events (292) compared to the gravity vector information (200).

\section{Discussion and conclusions}

It is known that high-resolution, one-point measurements of surface fluctuations collected by instruments such as downward-looking echo sounders generated by wake waves can be used for ship detection (Torsvik et al. 2015). Such measurements often require expensive field equipment that can be difficult to deploy and are negatively affected by wind waves. The data can be evaluated to estimate the sailing regime in terms of depth Froude numbers, speed, and distance to the measurement location to be estimated (Torsvik et al. 2015; Pethiyagoda et al. 2017, 2018).

The main goal of this work was to investigate the use of time series of wake waves for vessel detection using 
other technologies. We have demonstrated that information of comparable quality (i.e., suitable for the detection of properties of the ship and its sailing regime) can be produced using time series of properly located high-resolution pressure sensors. The use of such devices is on many occasions technically simpler, less expensive, and much less affected by atmospheric conditions. The spectrogram technique of windowed Fourier analysis is identical for the two approaches.

A specific feature of the use of the pressure signal is its attenuation for shorter waves in the water column. On the one hand, this feature may serve as a convenient low-pass filter to single out the ship wakes from the background of short-period wind seas. On the other hand, this feature may lead to an unacceptable level of low-frequency pressure fluctuations and/or noise in the recorded signal. We demonstrate that this shortcoming can be mitigated by using synchronized time series from an ensemble of pressure sensors in cases when the low-frequency pressure variations are incoherent. The resulting signatures of wakes in almost all cases match the corresponding references retrieved from the automatic identification system database.

The increased signal-to-noise ratio produced by overlapping spectrograms from several sensors apparently provides a simple and possibly better approach to the automatic detection of ship passages and several properties of her movement. The extended dataset, that also contains proxy information about near-bed velocities, could provide an additional basis for automatic vessel detection and may overcome some of the difficulties discussed in Torsvik et al. (2015). In particular, the use of multimodal datasets to characterize the properties of the wake has clear potential to increase the success rate of wake detection and quantification of the ship's sailing regime compared to the tracking of just one physical quantity from above the water level.

In vessel detection applications, the location, speed, and course of the vessel are the key features to be determined. The main message from the presented analysis is that spectrogram representations constructed using the pressure time series are of sufficient quality for the interpretation of the different components of ship wakes. The properties of these components (the slopes of the branches that correspond to diverging and transverse waves and the location of the intersection point of these branches) allow the quantification of several features of the vessel movement, such as the shortest distance of the sailing line to the measurement location, the location at which the first waves of the wake were produced, and the speed of the vessel and the local depth Froude number (Torsvik et al. 2015; Pethiyagoda et al. 2018) based on the evolution of a wake in time at a fixed point.

We also demonstrated that additional information provided by multisensor flow measurement devices can serve as a useful aid for the rapid detection of ship wakes. The proxy of near-bed water velocity from the described devices known as hydromasts (Ristolainen et al. 2016) may perform nearly as well as the procedure of detecting wakes from the spectrograms. The use of such datasets requires careful calibration and additional preprocessing tools to improve the accuracy and reliability of ship detection.

The improvement in the quality of spectrogram representation of the wakes using the signals from a cluster of closely positioned sensors is a first step toward the use of multiple sensor technology. The use of such clusters may serve as a feasible way toward the quantification of the vessel's course based on the analysis of phase shifts in single crests of the approaching wake. Another standing research question is the determination of the particular vessel (or vessel type) based on its wake. They both seem feasible using synchronized information in multimodal data streams from a properly positioned cluster of measurement devices.

In conclusion, we demonstrated that datasets from near-bed pressure sensors are equivalent to data of water surface elevation collected using surface measurements when considering, in the context of spectrogram technique, the detection of vessels and their sailing regimes. The use of synchronized multisensor systems makes it possible to substantially suppress noise and improve the quality of the outcome. Water movement data, which are also collected by the hydromast sensors, provide rapid detection of ship passages with a high success rate.

Acknowledgments. The current work was done in the frame of the European Union's Horizon 2020 research and innovation program project LAkHsMI under Grant Agreement 635568. Also, this research was supported by the Estonian Ministry of Education and Research (Estonian Research Council, Institutional Support IUT33-3 and PUT 1690) and the European Regional Development Fund program Mobilitas Pluss, reg.nr 2014-2020.4.01.16-0024, and cosupported by the Flag-ERA project FuturICT2.0, Estonian Center of Excellence EXCITE, the Estonian Research Infrastructures Roadmap object Infotechnological Mobility Observatory (IMO), and Baltic Research Programme (EEA Financial Mechanisms 2014-2021) project "Solutions to current and future problems on natural and constructed shorelines, eastern Baltic Sea" (EMP480). 


\section{REFERENCES}

Anupriya, K. R., and T. Sasilatha, 2018: Ship intrusion detection system-A review of the state of the art. Soft Computing Systems: ICSCS 2018, I. Zelinka et al., Eds., Communications in Computer and Information Science, Vol. 837, Springer, 147-154, https://doi.org/10.1007/978-981-13-1936-5_17.

Benassai, G., V. Piscopo, and A. Scamardella, 2015: Spectral analysis of waves produced by HSC for coastal management. J. Atmos. Oceanic Technol., 20, 417-428, https://doi.org/ 10.1007/S00773-014-0290-1.

BigOceanData, 2019: BigOceanData. Accessed 14 April 2019, http://www.bigoceandata.com/.

Bleckmann, H., and R. Zelick, 2009: Lateral line system of fish. Integr. Zool., 4, 13-25, https://doi.org/10.1111/j.17494877.2008.00131.x.

Cavaleri, L., 1980: Wave measurement using pressure transducer. Oceanol. Acta, 3, 339-346.

Chen, P. F., Y. M. Huang, J. M. Mou, and P. H. A. J. M. van Gelder, 2018: Ship collision candidate detection method: A velocity obstacle approach. Ocean Eng., 170, 186-198, https://doi.org/ 10.1016/j.oceaneng.2018.10.023.

Didenkulova, I., A. Sheremet, T. Torsvik, and T. Soomere, 2013: Characteristic properties of different vessel wake signals. J. Coastal Res., 65, 213-218, https://doi.org/10.2112/SI65-037.1.

Dugad, S., V. Puliyadi, H. Palod, N. Johnson, S. Rajput, and S. Johnny, 2016: Ship intrusion detection security system using HoG \& SVM. Int. J. Adv. Res. Comput. Eng. Technol., 5, 2504-2507, http://ijarcet.org/wp-content/uploads/IJARCETVOL-5-ISSUE-10-2504-2507.pdf.

Estonian Maritime Administration, 2019: Estonian Maritime Administration's web application Nutimeri. Accessed 3 May 2019, https://gis.vta.ee/nutimeri/.

Fang, M. C., R. Y. Yang, and I. V. Shugan, 2011: Kelvin ship wake in the wind waves field and on the finite sea depth.J. Mech., 27, 71-77, https://doi.org/10.1017/jmech.2011.9.

Fujino, I., C. Claramunt, and A.-O. Boudraa, 2018: Extracting courses of vessels from AIS data and real-time warning against off-course. Proc. Second Int. Conf. on Big Data Research, Weihai, China, Association for Computing Machinery, 62-69, https://doi.org/10.1145/3291801.3291823.

Gierull, C. H., 2019: Demystifying the capability of sublook correlation techniques for vessel detection in SAR imagery. IEEE Trans. Geosci. Remote Sens., 57, 2031-2042, https:// doi.org/10.1109/TGRS.2018.2870716.

Goda, Y., 2010: Reanalysis of regular and random breaking wave statistics. Coastal Eng. J., 52, 71-106, https://doi.org/10.1142/ S0578563410002129.

Huang, W., D. Wang, H. Garcia, O. R. Godø, and P. Ratilal, 2017: Continental shelf-scale passive acoustic detection and characterization of diesel-electric ships using a coherent hydrophone array. Remote Sens., 9, 772, https://doi.org/10.3390/ rs9080772.

Karimpour, A., and Q. Chen, 2017: Wind wave analysis in depth limited water using OCEANLYZ, a MATLAB toolbox. Comput. Geosci., 106, 181-189, https://doi.org/ 10.1016/j.cageo.2017.06.010.

Kuo, Y.-Y., and Y.-F. Chiu, 1994: Transfer function between wave height and wave pressure for progressive waves. Coastal Eng., 23, 81-93, https://doi.org/10.1016/0378-3839(94)90016-7.

Kurekin, A. A., B. R. Loveday, O. Clements, G. D. Quartly, P. I. Miller, G. Wiafe, and K. A. Agyekum, 2019: Operational monitoring of illegal fishing in Ghana through exploitation of satellite Earth observation and AIS data. Remote Sens., 11, 293, https://doi.org/10.3390/rs11030293.

Kuznetsov, N. G., V. G. Maz'ya, and B. Vainberg, 2002: Linear Water Waves: A Mathematical Approach. Cambridge University Press, 513 pp.

Leppäranta, M., and K. Myrberg, 2009: Physical Oceanography of the Baltic Sea. Springer, 378 pp.

Newman, J. N., 1977: Marine Hydrodynamics. MIT Press, 402 pp.

Panico, A., M. D. Graziano, and A. Renga, 2017: SAR-based vessel velocity estimation from partially imaged Kelvin pattern. IEEE Geosci. Remote Sens. Lett., 14, 2067-2071, https:// doi.org/10.1109/LGRS.2017.2751083.

Park, J.-J., S. Oh, K.-A. Park, P.-Y. Foucher, J.-C. Jang, M. Lee, T.-S. Kim, and W.-S. Kang, 2018: The ship detection using airborne and in-situ measurements based on hyperspectral remote sensing. J. Korean Earth Sci. Soc., 38, 535-545, https:// doi.org/10.5467/JKESS.2017.38.7.535.

Pethiyagoda, R., S. W. McCue, and T. J. Moroney, 2017: Spectrograms of ship wakes: Identifying linear and nonlinear wave signals. J. Fluid Mech., 811, 189-209, https:// doi.org/10.1017/jfm.2016.753.

_, T. J. Moroney, G. J. Macfarlane, J. R. Binns, and S. W. McCue, 2018: Time-frequency analysis of ship wave patterns in shallow water: Modelling and experiments. Ocean Eng., 158, 123-131, https://doi.org/10.1016/j.oceaneng.2018.01.108.

Rabaud, M., and F. Moisy, 2013: Ship wakes: Kelvin or Mach angle? Phys. Rev. Lett., 110, 214503, https://doi.org/10.1103/ PhysRevLett.110.214503.

Renga, A., M. D. Graziano, and A. Moccia, 2019: Segmentation of marine SAR images by sublook analysis and application to sea traffic monitoring. IEEE Trans. Geosci. Remote Sens., 57, 1463-1477, https://doi.org/10.1109/TGRS.2018.2866934.

Ristolainen, A., J. A. Tuhtan, A. Kuusik, and M. Kruusmaa, 2016: Hydromast: A bioinspired flow sensor with accelerometer. Biomimetic and Biohybrid Systems, N. Lepora et al., Eds., Lecture Notes in Computer Science, Vol. 9793, Springer, 510517, https://doi.org/10.1007/978-3-319-42417-0_55.

- _ — and M. Kruusmaa, 2019: Continuous, near-bed current velocity estimation using pressure and inertial sensing. IEEE Sens. J., 19, 12 398-12 406, https://doi.org/10.1109/ jsen.2019.2937954.

Sheremet, A., U. Gravois, and M. Tian, 2013: Boat-wake statistics at Jensen Beach, Florida. J. Waterw. Port Coastal Ocean Eng., 139, 286-294, https://doi.org/10.1061/(ASCE)WW.19435460.0000182 .

Siegert, G., J. Hoth, P. Banyś, and F. Heymann, 2019: Generic framework for vessel detection and tracking based on distributed marine radar image data. CEAS Space J., 11, 65-79, https://doi.org/10.1007/s12567-018-0208-6.

Soomere, T., 2005: Fast ferry traffic as a qualitatively new forcing factor of environmental processes in non-tidal sea areas: A case study in Tallinn Bay, Baltic Sea. Environ. Fluid Mech., 5, 293-323, https://doi.org/10.1007/s10652005-5226-1.

, 2007: Nonlinear components of ship wake waves. Appl. Mech. Rev., 60, 120-138, https://doi.org/10.1115/1.2730847.

_ K. E. Parnell, and I. Didenkulova, 2011: Water transport in wake waves from high-speed vessels. J. Mar. Syst., 88, 74-81, https://doi.org/10.1016/j.jmarsys.2011.02.011.

Sorensen, R. M., 1973: Ship-generated waves. Adv. Hydrosci., 9, 49-83, https://doi.org/10.1016/B978-0-12-021809-7.50007-9.

Till, G., 2013: Seapower: A Guide for the Twenty-First Century. Routledge, 432 pp., https://doi.org/10.4324/9780203880487. 
Torsvik, T., T. Soomere, I. Didenkulova, and A. Sheremet, 2015: Identification of ship wake structures by a time-frequency method. J. Fluid Mech., 765, 229-251, https://doi.org/10.1017/ jfm.2014.734.

Wehausen, J. V., 1973: The wave resistance of ships. Adv. Appl. Mech., 13, 93-245, https://doi.org/10.1016/S0065-2156(08)70144-3.

Wyatt, D. C., and R. E. Hall, 1988: Analysis of ship-generated surface waves using a method based upon the local Fourier transform. J. Geophys. Res., 93, 14133-14 164, https://doi.org/ 10.1029/JC093iC11p14133.
Zhu, C., H. Garcia, A. Kaplan, M. Schinault, N. O. Handegard, O. R. Godø, W. Huang, and P. Ratilal, 2018: Detection, localization and classification of multiple mechanized ocean vessels over continental-shelf scale regions with passive ocean acoustic waveguide remote sensing. Remote Sens., 10, 1699, https://doi.org/10.3390/rs10111699.

Zilman, G., A. Zapolski, and M. Marom, 2004: The speed and beam of a ship from its wake's SAR images. IEEE Trans. Geosci. Remote Sens., 42, 2335-2343, https://doi.org/10.1109/ TGRS.2004.833390. 